\title{
Eczema drugs tacrolimus (Protopic) and pimecrolimus (Elidel): cancer concerns
}

Published at www.cmaj.ca on Apr. 7, 2005.

Reason for posting: Many patients with eczema, or atopic dermatitis, are prescribed the topical immunomodulators tacrolimus and pimecrolimus. The drugs are often given to people for whom the potential side effects of topical corticosteroids (e.g., systemic absorption, skin thinning, telangiectasia) are a concern. However, the US Food and Drug Administration (FDA) recently reviewed the safety of these agents and warned that they may be associated with a risk of cancer. ${ }^{1}$

The drugs: Tacrolimus and pimecrolimus bind and inactivate calcineurin (a calcium- and calmodulin-dependent serine and threonine phosphatase) and may act by inhibiting T-lymphocyte activation, down-regulating numerous interleukins, interferon- $\gamma$, granulocyte-macrophage colonystimulating factor and tumour necrosis factor- $\alpha$, and affecting the function of mast cells, basophils and Langerhans cells.

Both agents are more effective than placebo in treating atopic dermatitis. Tacrolimus ( $0.03 \%$ and $0.1 \%$ preparations) is more effective than mild topical steroids, and the $0.1 \%$ preparation is as effective as more potent topical steroids. ${ }^{2}$ In con- trast, pimecrolimus is less effective than potent steroids $(0.1 \%$ betamethasone valerate), but its efficacy relative to mild corticosteroids is unclear. ${ }^{2}$

Common adverse effects include mild, local, temporary burning or pruritus, and users may have increased risk of local varicella-zoster virus infection, herpes simplex infection and eczema herpeticum. Children under the age of 2 receiving topical pimecrolimus had higher rates of respiratory tract infections than children receiving the placebo.

Lymphadenopathy, usually transient and related to underlying infections, has been reported. However, patients taking systemic tacrolimus (as an immunosuppressive agent after liver and organ transplantation) have reported lymphomas and solid organ tumours, possibly because their defences against cancer have been suppressed. Only small amounts of the drugs are usually absorbed through the skin; however, some children given topical tacrolimus have blood levels of the drug similar to those given its systemic form. ${ }^{1}$

Animals (mice, rats, monkeys) given high doses of the drugs topically or orally have a

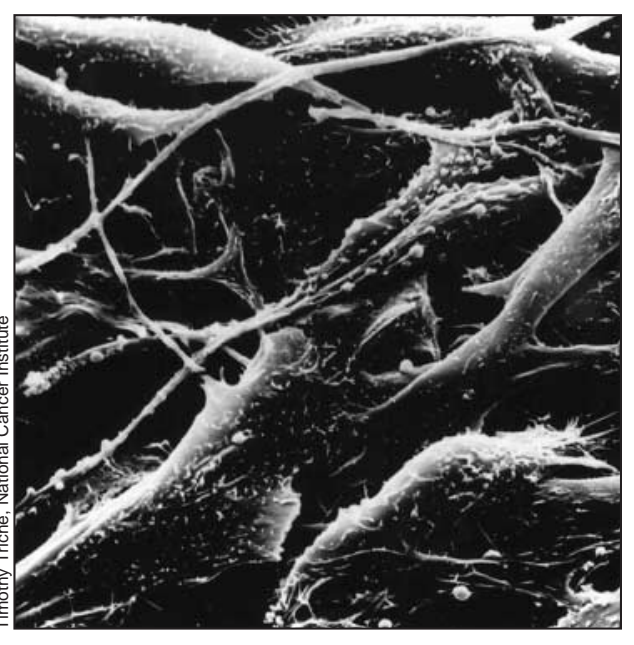

Melanoma

risk of cancer that is dependent on both the duration and dose of the drug. ${ }^{1,3}$ Long-term safety trials involving humans have not been done.

Causative associations are uncertain, but the FDA is also reporting the cases of several patients in whom cancer developed after drug use. For tacrolimus, 19 cases of cancer were reported, involving 16 adults and 3 children under the age of 16. The cancers were diagnosed 21-790 days after the start of therapy (the median time to diagnosis was 150 days). Nine cases involved lymphomas, and 10 involved skin

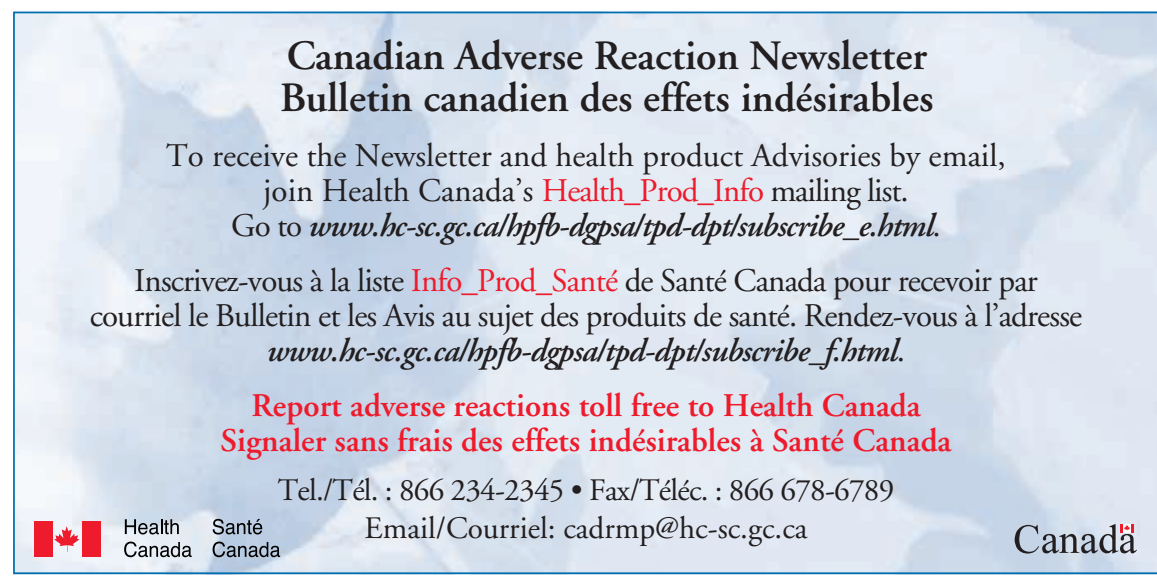


tumours ( 7 at the site of the drug application). Tumour types included squamous cell carcinoma, cutaneous sarcoma and malignant melanoma. For pimecrolimus, 10 postmarketing cases of cancer were reported, involving 4 children ( 3 less than 6 years of age) and 6 adults. Of the 10 cases, 6 involved cutaneous tumours and 4 were lymphomas. Diagnoses were made 7-300 days after treatment was started (median time to diagnosis was 90 days).

What to do: As second-line agents, these drugs should be used only if other therapies (topical corticosteroids, emollients) are ineffective or inap- propriate. They should not be used by patients with weakened or compromised immune systems, by children under the age of 2 or by patients with active viral skin infections. Short-term or intermittent use is advised. Unfortunately, atopic dermatitis is an uncomfortable, common and chronic condition. Patients should be warned of the potential cancer risk and carefully monitored clinically when taking the drugs. Any patient with nonresolving lymphadenopathy should be appropriately investigated. The lowest concentration of the drugs needed to control a patient's symptoms should be used. Unnecessary and potentially harmful ultraviolet expo- sure (from the sun and tanning beds) should be avoided.

Eric Wooltorton

Associate Editor, CMAJ

\section{References}

1. US Food and Drug Administration. FDA Public Health Advisory: Elidel (pimecrolimus) cream and Protopic (tacrolimus) ointment. 2005 Mar 10 Available at: www.fda.gov/medwatch /SAFETY/2005/safety05.htm\#Elidel (accessed 2005 Mar 30).

2. Ashcroft DM, Dimmock P, Garside R, Stein K, Williams HC. Efficacy and tolerability of topical pimecrolimus and tacrolimus in the treatment of atopic dermatitis: meta-analysis of randomized controlled trials. $B M \mathcal{F}$ 2005;330:516-22.

3. Niwa Y, Terashima T, Sumi H. Topical application of the immunosuppressant tacrolimus accelerates carcinogenesis in mouse skin. $\mathrm{Br} 7$ Dermatol 2003;149:960-7.

\section{IN THE LITERATURE}

\section{Does coronary revascularization before major vascular surgery decrease mortality?}

McFalls EO, Ward HB, Moritz TE, Goldman S, Krupski WC, Littooy $\mathrm{F}$, et al. Coronary-artery revascularization before elective major vascular surgery. N Engl J Med 2004;351(27):2795-804.

Background: Evidence gathered over the last 30 years has permitted stratification of patients undergoing noncardiac surgery into categories of high, intermediate and low cardiac risk $^{1-3}$ and clarified the effectiveness of medical interventions, princi perioperative complications. ${ }^{4}$ However, until now, the benefit of preoperative coronary revascularization (through percutaneous angioplasty or bypass surgery) has not been studied in

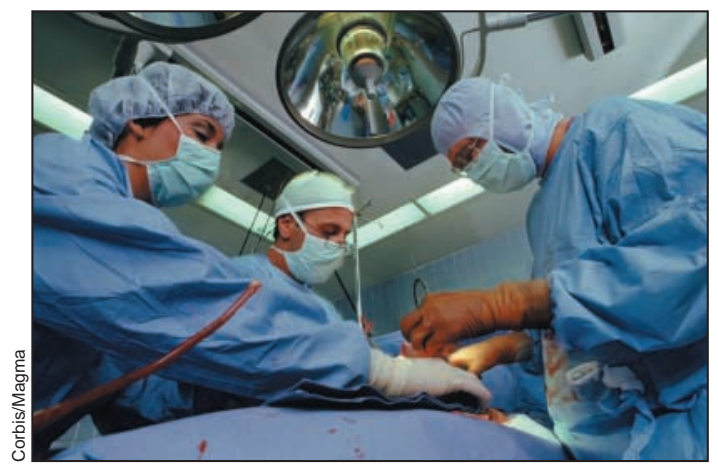

a randomized trial. Guidelines recommend conservative management for patients with stable coronary artery disease and lowrisk coronary anatomy, ${ }^{5}$ but practice variation occurs. This important addition to an evidence-based approach to the management of such patients.

Design: This study enrolled 510 patients from 18 US Veterans Affairs medical centres (98\% male) between 1997 and 2003 . Subjects were scheduled for elective vascular surgery $(33 \%$ for an expanding abdominal aneurysm, $67 \%$ for symptomatic arterial occlusive disease of the legs). To receive cardiac catheterization, patients had to be deemed at increased risk of perioperative cardiac complications by a cardiologist. Those who had angiographic evidence of stenosis greater than $70 \%$ in at least 1 coronary artery were elirandomized trial is therefore an gible. Exclusion criteria included severe coexisting illness, previous revascularization without evidence of recurrent ischemia, stenosis greater than $50 \%$ of the left main coronary artery, a left ventricular ejection fraction of less than $20 \%$ and severe aortic stenosis. Patients were randomly assigned to coronary artery revascularization (CAR) before surgery or to no revascularization. Percutaneous coronary intervention was performed on $59 \%$ of the patients and coronary artery bypass surgery on $41 \%$. The primary end point was long-term mortality, with a minimum followup of 1 year and a median follow-up of slightly over 2.5 years.

Results: Of the study patients, $74 \%$ demonstrated a moderate or large reversible defect on stress imaging or were considered to be at intermediate or high cardiac risk according to the criteria of Eagle $^{5}$ or Lee and associates. $^{2}$ Before vascular surgery, there were 10 deaths in the CAR group and 1 death in 